\title{
Genetics and Periodontal Disease
}

Thomas Michael Hassell

Professor of Periodontics, University of Washington, Seattle, and Director of Clinical Research, Optiva Co., Bellevue, WA

For decades dentists have debated the relative roles of heredity and environmental factors ("nature vs. nurture") in the pathogenesis of diseases of the periodontium. Definitive conclusions have been difficult to achieve because of the complexity of the interactions that occur between host-response mechanisms and bacterial pathogens. Thus, for over 30 years, dental scientists have concentrated their research efforts on elucidating the microbiological etiologic factors of periodontitis, rather than studying host susceptibility.

During the last several years, however, there has been a rebirth of interest in the search for hereditary factors that could determine periodontal disease susceptibility. This has been made possible by 1) modern techniques of genetic and statistical analysis, 2) in vitro methodologies for examining cellular responses to pathogenic substances, 3) exploitation of the human twin paradigm for clinical investigations, 4) identification of pro-inflammatory cytokines such as interleukin-1 (IL-1), and 5) the discovery of several genetic polymorphisms in the genes that code for IL-1.

In this presentation, three lines of evidence that indicate a specific role of the genotype in periodontal disease susceptibility will be discussed. New diagnostic tests will be described, which can rapidly, reliably and inexpensively identify persons who are susceptible. Recent scientific evidence demonstrates that up to 30 percent of the population is genetically susceptible to periodontitis.

Thus, individuals who test positive for the "susceptibility" genotype of periodontitis can be subjected to elevated preventive measures including more rigorous home care as well as more frequent professional care, to prevent periodontal destruction. 